\title{
The relation of anthropometric measurements and insulin resistance in patients with polycystic kidney disease
}

\author{
Bennur Esen ${ }^{1}$, Emel Sağlam Gokmen², Mahmut Kaya ${ }^{3}$, Burak Ozkan4, \\ Ahmet Engin Atay ${ }^{2}$ \\ 'Department of Nephrology, Acıbadem Taksim Hospital, Istanbul, Turkey; \\ ${ }^{2}$ Bagcilar Education and Research Hospital, Internal Medicine, Istanbul, Turkey; \\ ${ }^{3}$ Department of Internal Medicine, School of Medicine, Yeni Yuzyil University, Istanbul, Turkey; \\ ${ }^{4}$ Department of Urology, School of Medicine, Acibadem University, Istanbul, Turkey
}

Address for Correspondence: Dr. Bennur Esen, Department of Nephrology, Acıbadem Taksim Hospital, Istanbul, Turkey.

Email: bennuresen@yahoo.com

\begin{tabular}{|l|}
\hline Access this article online \\
\hline $\begin{array}{l}\text { Website: } \\
\text { www.intern-med.com }\end{array}$ \\
\hline DOI: \\
10.1515/jtim-2016-0029 \\
\hline Quick Response Code: \\
\hline \\
\\
\\
\\
回 \\
\hline
\end{tabular}

\section{ABSTRACT}

Objective: To examine the frequency of insulin resistance (IR) and its relation with anthropometric measurements in patients with autosomal dominant polycystic kidney disease (ADPKD). Material and Methods: Nonobese 82 patients with ADPKD and 58 age matched healthy controls were enrolled into the study. None of participants were diabetic or receiving renal replacement therapies (RRT). IR was determined by homeostasis model assessment of insulin resistance (HOMA-IR) formula. Tanita body composition analyzer was used for anthropometric measurements. Creatinine clearance of participant were assessed by the modification of diet in renal diseases (MDRD). Results: Patients with ADPKD had significantly higher level of urea and creatinine, microalbuminuria, and lower level of MDRD. Body fat distribution and HOMA-IR in both the groups were similar. Systolic and diastolic blood pressure of patients were higher than those of controls. Conclusion: We failed to determine a higher frequency of IR among patients with ADPKD.

Key words: polycystic kidney disease, insulin resistance, anthropometric measurements, obesity, diabetes mellitus

\section{INTRODUCTION}

Autosomal dominant polycystic kidney disease (ADPKD) is the most common hereditary kidney disease which is associated with progressive deterioration of renal functions. ${ }^{[1]}$ End-stage renal disease (ESRD) is observed in $25 \%$ of patients aged $<50$ years and $50 \%$ of patients aged $<60$ years. ${ }^{[2]}$ Insulin resistance (IR) accompanies diabetes mellitus (DM), hypertension, dyslipidemia, glucose intolerance, and hyperuricemia in metabolic and cardiovascular disorders (CVDs). ${ }^{[3]}$ There are controversial reports in terms of IR in patients with ADPKD. ${ }^{[4,5]}$

Obesity is a risk factor for IR; however, quantity and distribution of body fat are important determinants of obesity-related risk factors. ${ }^{[6]}$ Abdominal or visceral fat is associated with higher incidence of IR and cardiovascular (CV) problems. ${ }^{[7]}$
Anthropometry is a term used to define the measurements of specific body segments or regions. Anthropometric measurements including height, weight, body mass index (BMI), and body fat distribution provide valuable data regarding the future risk of diabetes and CVDs. ${ }^{[8]}$

We aimed to examine the relation of IR and anthropometric measurements in patients with ADPKD.

\section{PATIENTS AND METHODS}

\section{Patients}

Eighty two patients with ADPKD admitted to Nephrology outpatients service of Bagcilar Education and Research Hospital between February and April 2015 were enrolled. Fifty eight age, gender, and BMI matched healthy individuals, including 25 males and 33 females, were assigned to 
control group. Exclusion criteria were DM, pregnancy, malignancy, additional chronic disorder, or patients receiving renal replacement therapies (RRT). None of the participants were under special dietary restrictions. The participants underwent physical examination, and demographic features of the participants were recorded. All the subjects read and signed the informed consent forms before enrolling into the study. The ethics committee of the Bagcilar Education and Research Hospital approved the study.

\section{Methods}

Blood samples were collected after a 12 -h fasting period. Biochemical variables including serum glucose, urea, creatinine, uric acid, aspartate aminotransferase (AST), alanine aminotransferase (ALT), lactate dehydrogenase (LDH), calcium, sodium, potassium, total protein, albumin, total cholesterol, low-density lipoprotein (LDL), high-density lipoprotein (HDL), and triglyceride were analyzed by photometric method on the Siemens Advia 1800 device (Siemens Healthcare Diagnostics, Kobe, Japan). Hormone parameters including HbA1c, insulin, and thyroid-stimulating hormone (TSH) were analyzed by chemiluminescence immunoassay method on a Siemens Advia Centaur device (XE-5000, Sysmex Corp., Kobe, Japan). Urine analysis was performed by spectrophotometric method on a Siemens Advia 1800 device (XE-5000, Sysmex Corp., Kobe, Japan). Hemogram was performed in blood samples that were taken into tubes with ethylenediaminetetraacetic acid using an automatic blood counter (XE-5000; Sysmex Corp, Kobe, Japan). Biochemical and hormonal parameters were examined simultaneously in this study.

Insulin resistance was determined by homeostasis model assessment of insulin resistance (HOMA-IR) formula as defined by Matthews et al. ${ }^{\left[{ }^{[9]}\right.}$ The normal value for HOMAIR was considered to be 2.1-2.5. HOMA-IR value of $>2.5$ was considered glucose intolerance and DM. The following formula was used to determine HOMA-IR: HOMA-IR=
[Fasting plasma insulin $(\mathrm{U} / \mathrm{mL}) \times$ Fasting blood glucose $(\mathrm{mg} / \mathrm{dL})] / 405$; HOMA-IR $=[$ Fasting plasma insulin $(\mathrm{U} /$ $\mathrm{mL}) \times$ Fasting blood glucose $(\mathrm{mmol} / \mathrm{L})] / 22.5$.

Glomerular filtration rate was measured by the modification of diet in renal disease (MDRD) formula (Glomerular filtration rate: $186 \times$ Serum creatinine $-1.154 \times$ age -0.203 $\times$ genderrace).

Height and weight of the participants were measured using Tanita Body Composition Analyzer (Tanita Corporation of America, IL, USA). BMI was calculated as weight/ height ${ }^{2}\left(\mathrm{~kg} / \mathrm{m}^{2}\right)$.

Urine albumin excretion rate exceeding $20 \mu \mathrm{g} / \mathrm{min}$ (30 $\mathrm{mg} /$ day), in the absence of uncontrolled hypertension or urinary tract infection, was defined as microalbuminuria.

\section{Statistical analysis}

SPSS 22.0 (SPSS, Chicago, Illinois, USA) package program was used for statistical analysis. Parametric variables were compared with independent $t$-test, ordinal data were compared with Mann Whitney U test, and non-parametric variables were compared with chi-square test. Quantitative parameters were compared with Kruskal-Wallis test. Spearman's rho correlation test was used to evaluate the relation of parametric variables. A $P$ value of $<0.05$ was considered significant.

\section{RESULTS}

A total of 82 patients with ADPKD, 34 male and 48 female, and 58 healthy subjects, 25 male and 33 female, as controls were enrolled into the study. Demographic properties of study group were shown in Table 1.

Patients group had significantly higher level of urea, creatinine, and uric acid when compared to controls $(P=0.001, P=0.001$, and $P=0.002$; respectively). Patients had significantly lower MDRD and urine density $(P=0.001$ and $P=0.022$, respectively).

\begin{tabular}{|c|c|c|c|}
\hline & Patients & Controls & ${ }^{\mathrm{a} P}$ \\
\hline & Ort \pm SS & Ort \pm SS & \\
\hline Age (years) & $47.71 \pm 15.32$ & $46.69 \pm 6.74$ & 0.595 \\
\hline Height (cm) & $163.73 \pm 9.39$ & $163.9 \pm 10.48$ & 0.924 \\
\hline Weight (cm) & $74.77 \pm 13.37$ & $71.65 \pm 8.76$ & 0.103 \\
\hline \multirow[t]{2}{*}{ BMI $\left(\mathrm{kg} / \mathrm{m}^{2}\right)$} & $27.95 \pm 5.19$ & $26.71 \pm 2.03$ & 0.056 \\
\hline & $n(\%)$ & $n(\%)$ & ${ }^{\mathrm{b}} \boldsymbol{P}$ \\
\hline \multicolumn{4}{|l|}{ Gender } \\
\hline Male & $34(41.5)$ & $25(43.1)$ & 0.847 \\
\hline Female & $48(58.5)$ & $33(56.9)$ & \\
\hline
\end{tabular}

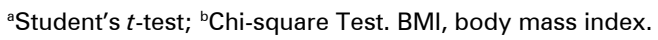


On the other hand, proteinuria and microalbuminuria-tocreatinine ratio of patients were significantly higher $(P=0.004$ and $P=0.001$, respectively) (Table 2 ).

Body fat and liquid distribution of groups were similar $(P$ $>0.05$ ) (Table 3).

The mean systolic and diastolic blood pressure of male patients were significantly higher than that of controls
$(P=0.013, P<0.05 ; P=0.007, P<0.01)$. Male controls had significantly higher MDRD when compared to male patients $(P=0.001, P<0.01)$. Male patients had significantly higher level of urea, creatinine, and uric acid and higher microalbuminuria-to-creatinine ratio $(P=0.018$, $P=0.001, P=0.029$, and $P=0.005$, respectively). Urine density and mean body impedance of male patients were lower than those of male controls $(P=0.005$ and $P=$ 0.046 , respectively) (Table 4)

\begin{tabular}{|c|c|c|c|}
\hline & Patients & Controls & $P$ \\
\hline & Mean \pm SD (Median) & Mean \pm SD (Median) & $r$ \\
\hline Systolic blood pressure $(\mathrm{mmHg})$ & $123.66 \pm 12.93(120.00)$ & $121.79 \pm 12.72(120.00)$ & ${ }^{\mathrm{a}} \mathbf{0 . 6 8 8}$ \\
\hline Diastolic blood pressure $(\mathrm{mmHg})$ & $76.32 \pm 80.00(80.00)$ & $75.17 \pm 9.50(75.00)$ & ${ }^{\mathrm{a}} 0.672$ \\
\hline MDRD & $91.26 \pm 45.26$ & $121.09 \pm 27.95$ & ${ }^{\mathrm{b}} 0.001 * *$ \\
\hline HOMA-IR ( $\mu \mathrm{U} / \mathrm{mmol})$ & $2.89 \pm 1.88(2.58)$ & $2.59 \pm 1.84(1.97)$ & ${ }^{\mathrm{a}} 0.209$ \\
\hline HbA1c (\%) & $5.53 \pm 0.39$ & $5.49 \pm 0.40$ & ${ }^{\mathrm{b}} 0.627$ \\
\hline Glucose (mg/dL) & $95.93 \pm 10.41$ & $93.93 \pm 9.43$ & ${ }^{\mathrm{b}} 0.246$ \\
\hline Urea $(\mathrm{mg} / \mathrm{dL})$ & $42.79 \pm 25.05(33.55)$ & $27.49 \pm 8.19(26.20)$ & ${ }^{\mathrm{a}} 0.001 *$ \\
\hline Creatinine (mg/dL) & $1.23 \pm 0.95(0.93)$ & $0.73 \pm 0.15(0.72)$ & ${ }^{\mathrm{a}} 0.001 * *$ \\
\hline Uric acid (mg/dL) & $5.23 \pm 1.73$ & $4.49 \pm 1.05$ & ${ }^{\mathrm{b}} 0.002 * *$ \\
\hline AST (U/L) & $18.26 \pm 7.48(17.00)$ & $19.11 \pm 6.16(17.00)$ & ${ }^{\mathrm{a}} 0.172$ \\
\hline ALT (U/L) & $16.25 \pm 7.82(14.00)$ & $19.27 \pm 10.76(17.00)$ & ${ }^{\mathrm{a}} \mathbf{0 . 0 7 1}$ \\
\hline LDH (U/L) & $190.90 \pm 72.63(186.00)$ & $194.95 \pm 29.07(187.00)$ & ${ }^{\mathrm{a}} \mathbf{0 . 0 7 8}$ \\
\hline $\mathrm{TG}(\mathrm{mg} / \mathrm{dL})$ & $139.38 \pm 74.18(128.00)$ & $140.45 \pm 96.41(108.00)$ & ${ }^{\mathrm{a}} 0.476$ \\
\hline Total cholesterol (mg/dL) & $201.31 \pm 48.76$ & $194.98 \pm 44.33$ & ${ }^{\mathrm{b}} 0.436$ \\
\hline HDL-c (mg/dL) & $53.19 \pm 12.98$ & $52.93 \pm 16.31$ & ${ }^{\mathrm{b}} 0.916$ \\
\hline LDL-c (mg/dL) & $126.34 \pm 42.61$ & $116.20 \pm 37.13$ & ${ }^{\mathrm{b}} 0.148$ \\
\hline $\mathrm{Ca}(\mathrm{mg} / \mathrm{dL})$ & $9.50 \pm 0.57$ & $9.43 \pm 0.51$ & ${ }^{\mathrm{b}} 0.449$ \\
\hline $\mathrm{Na}(\mathrm{mEq} / \mathrm{L})$ & $140.23 \pm 15.60(142)$ & $141.14 \pm 2.07(141)$ & ${ }^{\mathrm{a}} 0.055$ \\
\hline$K(m E q / L)$ & $4.51 \pm 0.44$ & $4.42 \pm 0.41$ & ${ }^{\mathrm{b}} 0.177$ \\
\hline Total protein $(\mathrm{g} / \mathrm{dL})$ & $7.33 \pm 0.51$ & $7.27 \pm 0.39$ & ${ }^{\mathrm{b}} 0.482$ \\
\hline Albumin $(\mathrm{g} / \mathrm{dL})$ & $4.54 \pm 0.37$ & $4.58 \pm 0.28$ & ${ }^{\mathrm{b}} \mathbf{0 . 5 2 7}$ \\
\hline TSH (mU/L) & $2.09 \pm 1.26(1.75)$ & $2.72 \pm 2.75(2)$ & ${ }^{\mathrm{a}} \mathbf{0 . 3 6 7}$ \\
\hline Insulin (mU/L) & $12.03 \pm 7.53(11.03)$ & $11.08 \pm 7.93(8.79)$ & ${ }^{\mathrm{a}} 0.232$ \\
\hline WBC $\left(/ \mathrm{mm}^{3}\right)$ & $7.12 \pm 1.85(7.06)$ & $7.25 \pm 2.20(6.97)$ & ${ }^{\mathrm{a}} 0.929$ \\
\hline HGB (g/dL) & $13.49 \pm 1.77$ & $13.89 \pm 1.66$ & ${ }^{\mathrm{b}} 0.172$ \\
\hline НСТ (\%) & $41.46 \pm 5.11$ & $42.64 \pm 4.57$ & ${ }^{\mathrm{b}} 0.164$ \\
\hline PLT $\left(/ \mathrm{mm}^{3}\right)$ & $241.29 \pm 62.38$ & $249.86 \pm 59.89$ & ${ }^{\mathrm{b}} \mathbf{0 . 4 1 7}$ \\
\hline MPV (fL) & $7.87 \pm 1.31$ & $8.16 \pm 1.80$ & ${ }^{\mathrm{b}} 0.267$ \\
\hline Urine density $\left(\mathrm{kg} / \mathrm{cm}^{3}\right)$ & $1013.24 \pm 7.19$ & $1015.93 \pm 6.11$ & ${ }^{\mathrm{b}} 0.022 *$ \\
\hline Urine protein $(\mathrm{mg} / \mathrm{dL})$ & $0.29 \pm 0.66(0)$ & $0.02 \pm 0.14(0)$ & ${ }^{\mathrm{a}} 0.004 * *$ \\
\hline Spot creatinine $(\mathrm{mg} / \mathrm{dL})$ & $131.19 \pm 76.35$ & $150.13 \pm 72.03$ & ${ }^{\mathrm{b}} 0.141$ \\
\hline Urine MA/KR (mg/g) & $90.9 \pm 174.6(17.5)$ & $6.80 \pm 7.56(3.95)$ & ${ }^{\mathrm{a}} 0.001 * *$ \\
\hline Urine microalbumin (mg/day) & $8.41 \pm 13.53(2.42)$ & $1.10 \pm 1.73(0.52)$ & ${ }^{\mathrm{a}} 0.001 * *$ \\
\hline Fat $(\mathrm{kg})$ & $20.97 \pm 10.65$ & $19.46 \pm 6.02$ & ${ }^{\mathrm{b}} 0.229$ \\
\hline Fat $(\%)$ & $27.16 \pm 11.22$ & $27.42 \pm 8.43$ & ${ }^{\mathrm{b}} 0.879$ \\
\hline Body impedance (ohm) & $546.21 \pm 79,93$ & $573.72 \pm 59.67$ & ${ }^{\mathrm{b}} 0.030 *$ \\
\hline Body muscle (kg) & $28.35 \pm 5.35$ & $27.81 \pm 4.79$ & ${ }^{\mathrm{b}} 0.542$ \\
\hline Body fat (kg) & $10.41 \pm 5.52$ & $9.96 \pm 3.27$ & ${ }^{\mathrm{b}} 0.556$ \\
\hline Metabolic age (years) & $49.19 \pm 16.7$ & $45.40 \pm 8.15$ & ${ }^{\mathrm{b}} 0.084$ \\
\hline Liquid $(\mathbf{k g})$ & $39,28 \pm 7.09$ & $38.09 \pm 7.21$ & ${ }^{\mathrm{b}} 0.339$ \\
\hline Liquid (\%) & $53.29 \pm 8.23$ & $53.09 \pm 6.15$ & ${ }^{\mathrm{b}} \mathbf{0 . 8 7 6}$ \\
\hline
\end{tabular}

a Mann-Whitney $U$ test; ${ }^{b}$ Student's $t$-test; ${ }^{*} P<0.05 ;{ }^{*} P<0.01$. MDRD, modification of diet in renal diseases; HOMA-IR, homeostasis model assessment of insulin resistance; AST, aspartate aminotransferase; ALT, alanine aminotransferase; LDH, lactate dehydrogenase; TG, triglycerides; HDL-C, high-density lipoprotein cholesterol; LDL-c, low-density lipoprotein cholesterol; TSH, thyroid-stimulating hormone; WBC, white blood cell count; HGB, hemoglobin; HCT, hematocrit; PLT, platelet; MPV, mean platelet volume. 
Esen $e t$ al.: The relation of anthropometric measurements and insulin resistance in patients with polycystic kidney disease

\begin{tabular}{|c|c|c|c|c|}
\hline & & Patients n (\%) & Controls n (\%) & $P$ \\
\hline \multirow[t]{3}{*}{ Fat } & $<20 \%$ & $22(28.2)$ & $12(21.1)$ & 0.559 \\
\hline & $20-27 \%$ & $16(20.5)$ & $15(26.3)$ & \\
\hline & $>\mathbf{2 7} \%$ & $40(51.3)$ & $30(52.6)$ & \\
\hline \multirow[t]{3}{*}{ Liquid } & $<50 \%$ & 27 (34.6) & $23(40.4)$ & 0.738 \\
\hline & $50-60 \%$ & $34(43.6)$ & $24(42.1)$ & \\
\hline & $>60 \%$ & $17(21.8)$ & $10(17.5)$ & \\
\hline
\end{tabular}

Chi-square test; Body fat ratio: $<20 \%$ low, 20-27\% normal, $>27 \%$ high; Body liquid ratio: $<50 \%$ low, $50-60 \%$ normal, $>60 \%$ high.

\begin{tabular}{|c|c|c|c|}
\hline \multirow[t]{2}{*}{ Male participants } & Patients & Controls & \multirow{2}{*}{$P$} \\
\hline & Mean \pm SD (Median) & Mean \pm SD (Median) & \\
\hline Systolic blood pressure (mmHg) & $125.59 \pm 10.85(125.00)$ & $117.00 \pm 11.99(120.00)$ & ${ }^{\mathrm{a}} 0.013 *$ \\
\hline Diastolic blood pressure $(\mathrm{mmHg})$ & $76.41 \pm 5.52(80.00)$ & $71.20 \pm 8.81(70.00)$ & ${ }^{\mathrm{a}} 0.007 * *$ \\
\hline MDRD & $72.19 \pm 29.67$ & $100.51 \pm 16.31$ & ${ }^{\mathrm{b}} 0.001 *$ \\
\hline HOMA-IR ( $\mu \mathrm{U} / \mathrm{mmol})$ & $2.95 \pm 1.97(2.48)$ & $2.77 \pm 1.93(2.38)$ & ${ }^{\mathrm{a}} 0.742$ \\
\hline HbA1c (\%) & $5.49 \pm 0.35$ & $5.52 \pm 0.39$ & ${ }^{\mathrm{b}} \mathbf{0 . 7 3 4}$ \\
\hline Glucose (mg/dL) & $95.65 \pm 11.25$ & $93.88 \pm 8.29$ & ${ }^{\mathrm{b}} 0.509$ \\
\hline Urea $(\mathrm{mg} / \mathrm{dL})$ & $45.21 \pm 26.71(33.75)$ & $30.8 \pm 9.02(29.00)$ & ${ }^{\mathrm{a}} 0.018 *$ \\
\hline Creatinine (mg/dL) & $1.44 \pm 1.11(1.08)$ & $0.85 \pm 0.13(0.83)$ & ${ }^{\mathrm{a}} 0.001 *$ \\
\hline Uric acid (mg/dL) & $5.64 \pm 1.16$ & $5.01 \pm 0.96$ & ${ }^{\mathrm{b}} 0.029 *$ \\
\hline AST (U/L) & $18.38 \pm 8.39(17.00)$ & $19.74 \pm 5.28(18.00)$ & ${ }^{\mathrm{a}} 0.131$ \\
\hline ALT (U/L) & $17.21 \pm 7.12(15.50)$ & $22.45 \pm 12.99(19.30)$ & ${ }^{\mathrm{a}} 0.093$ \\
\hline LDH (U/L) & $201.38 \pm 107.66(180.00)$ & $199.92 \pm 29.13(188.00)$ & ${ }^{\mathrm{a}} \mathbf{0 . 0 7 6}$ \\
\hline TG (mg/dL) & $146.91 \pm 84.68(127.50)$ & $163.52 \pm 98.42(129.00)$ & ${ }^{\mathrm{a}} \mathbf{0 . 5 7 0}$ \\
\hline Total cholesterol (mg/dL) & $185.64 \pm 38.75$ & $191.76 \pm 46.72$ & ${ }^{\mathrm{b}} 0.585$ \\
\hline HDL-c (mg/dL) & $49.39 \pm 11.98$ & $45.01 \pm 11.24$ & ${ }^{\mathrm{b}} 0.160$ \\
\hline LDL-c (mg/dL) & $116.05 \pm 32.79$ & $117.44 \pm 38.75$ & ${ }^{\mathrm{b}} \mathbf{0 . 8 8 2}$ \\
\hline $\mathrm{Ca}(\mathrm{mg} / \mathrm{dL})$ & $9.45 \pm 0.58$ & $9.60 \pm 0.47$ & ${ }^{\mathrm{b}} 0.282$ \\
\hline $\mathrm{Na}(\mathrm{mEq} / \mathrm{L})$ & $143.21 \pm 3.47(143)$ & $142.20 \pm 2.18(142)$ & ${ }^{\mathrm{a}} 0.193$ \\
\hline$K(m E q / L)$ & $4.61 \pm 0.45$ & $4.42 \pm 0.40$ & ${ }^{\mathrm{b}} 0.104$ \\
\hline Total protein $(\mathrm{g} / \mathrm{dL})$ & $7.33 \pm 0.60$ & $7.41 \pm 0.40$ & ' 0.567 \\
\hline Albumin (g/dL) & $4.64 \pm 0.41$ & $4.70 \pm 0.21$ & ' 0.455 \\
\hline TSH (mU/L) & $2.21 \pm 1.57(1.84)$ & $1.94 \pm 1.03(1.76)$ & a 1.000 \\
\hline Insulin (mU/L) & $12.28 \pm 7.81(11.20)$ & $11.71 \pm 7.74$ (9.79) & ${ }^{\mathrm{a}} \mathbf{0 . 7 3 6}$ \\
\hline WBC $\left(/ \mathrm{mm}^{3}\right)$ & $7.37 \pm 1.97(7.18)$ & $7.58 \pm 2.26(6.95)$ & ${ }^{\mathrm{a}} 0.927$ \\
\hline HGB (g/dL) & $14.67 \pm 1.76$ & $15.31 \pm 1.06$ & ${ }^{\mathrm{b}} 0.110$ \\
\hline НСТ (\%) & $44.87 \pm 4.88$ & $46.53 \pm 2.67$ & ${ }^{\mathrm{b}} 0.129$ \\
\hline PLT $\left(/ \mathrm{mm}^{3}\right)$ & $228.66 \pm 54.41$ & $238.04 \pm 58.72$ & ${ }^{\mathrm{b}} 0.529$ \\
\hline MPV (fL) & $7.49 \pm 1.14$ & $7.86 \pm 1.64$ & ${ }^{\mathrm{b}} 0.309$ \\
\hline Urine density $\left(\mathrm{kg} / \mathrm{cm}^{3}\right)$ & $1013.15 \pm 6.29$ & $1017.68 \pm 5.34$ & ${ }^{\mathrm{b}} 0.005 * *$ \\
\hline Urine protein $(\mathrm{mg} / \mathrm{dL})$ & $0.35 \pm 0.75(0)$ & $0.05 \pm 0.22(0)$ & ${ }^{\mathrm{a}} \mathbf{0 . 0 7 7}$ \\
\hline Spot creatinine $(\mathrm{mg} / \mathrm{dL})$ & $143.02 \pm 78.22$ & $174.82 \pm 71.83$ & ${ }^{\mathrm{b}} 0.116$ \\
\hline Urine MA/KR $(\mathrm{mg} / \mathrm{g})$ & $91.65 \pm 171.26(17.50)$ & $8.14 \pm 9.46(4.90)$ & ${ }^{\mathrm{a}} 0.001 * *$ \\
\hline Urine microalbumin (mg/day) & $8.06 \pm 11.8(2.72)$ & $1.51 \pm 2.42(0.68)$ & ${ }^{\mathrm{a}} 0.002 * *$ \\
\hline Obesity degree (\%) & $16.19 \pm 11.24(14.83)$ & $11.85 \pm 5.35(10.85)$ & ${ }^{\mathrm{a}} 0.181$ \\
\hline Fat $(\mathbf{k g})$ & $16.16 \pm 9.22$ & $15.21 \pm 4.70$ & ${ }^{\mathrm{b}} 0.611$ \\
\hline Fat $(\%)$ & $19.62 \pm 9.13$ & $19.53 \pm 5.45$ & ${ }^{\mathrm{b}} 0.959$ \\
\hline Body impedance (ohm) & $507.18 \pm 62.91$ & $538.17 \pm 47.34$ & ${ }^{\mathrm{b}} 0.046 *$ \\
\hline Body muscle (kg) & $32.01 \pm 5.32$ & $32.46 \pm 3.51$ & ' 0.721 \\
\hline Body fat (kg) & $9.16 \pm 5.17$ & $9.35 \pm 3.33$ & ${ }^{\mathrm{b}} \mathbf{0 . 8 6 0}$ \\
\hline Metabolic age (years) & $47.29 \pm 17.10$ & $42.33 \pm 9.24$ & ${ }^{\mathrm{b}} 0.161$ \\
\hline Liquid (kg) & $45.28 \pm 5.80$ & $45.39 \pm 4.84$ & ${ }^{\mathrm{b}} 0.939$ \\
\hline Liquid (\%) & $58.81 \pm 6.69$ & $58.86 \pm 3.98$ & ${ }^{\mathrm{b}} 0.975$ \\
\hline
\end{tabular}

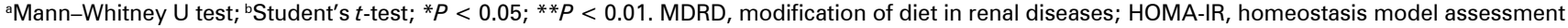
of insulin resistance; AST, aspartate aminotransferase; ALT, alanine aminotransferase; LDH, lactate dehydrogenase; TG, triglycerides; HDL-C, high-density lipoprotein cholesterol; LDL-c, low-density lipoprotein cholesterol; TSH, thyroid-stimulating hormone; WBC, white blood cell count; HGB, hemoglobin; HCT, hematocrit; PLT, platelet; MPV, mean platelet volume. 
The mean MDRD, urea, creatinine, and uric acid of female control subjects were significantly higher than those of patients group ( $P=0.001$ for all). Female patients had significantly higher proteinuria and microalbuminuria-tocreatinine ratio $(P=0.022$ and $P=0.001$, respectively). Similarly, female patients had higher mean obesity degree and body liquid mass $(P=0.017$ and $P=0.008$, respectively). There was no significant difference between female patients and controls in terms of systolic blood pressure, diastolic blood pressure, HOMA-IR, AST, ALT, $\mathrm{LDH}$, triglyceride, $\mathrm{Na}$, TSH, insulin, and leukocyte count $(P>0.05)$. We failed to demonstrate a significant difference between female patients and controls with regard to HbA1C, glucose, total cholesterol, HDL, LDL, Ca, K, total protein, albumin, hemoglobin, hematocrit, platelet, mean platelet volume, urine density, spot creatinine, fat mass, fat ratio, body muscle mass, body fat mass, metabolic age, and liquid ratio $(P>0.05)$ (Table 5).

\begin{tabular}{|c|c|c|c|}
\hline \multirow[t]{2}{*}{ Female participants } & \multirow{2}{*}{$\begin{array}{l}\text { Patients } \\
\text { Mean } \pm \text { SD (Median) }\end{array}$} & \multirow{2}{*}{$\begin{array}{l}\text { Controls } \\
\text { Mean } \pm \text { SD (Median) }\end{array}$} & \multirow{2}{*}{$P$} \\
\hline & & & \\
\hline Systolic blood pressure $(\mathrm{mmHg})$ & $122.29 \pm 14.18(120.00)$ & $125.42 \pm 12.21(125.00)$ & ${ }^{\mathrm{a}} 0.118$ \\
\hline Diastolic blood pressure $(\mathrm{mmHg})$ & $79.94 \pm 12.29(72.50)$ & $78.18 \pm 9.14(80.00)$ & a 0.152 \\
\hline MDRD & $104.77 \pm 49.62$ & $136.68 \pm 24.71$ & ${ }^{\mathrm{b}} 0.001 * *$ \\
\hline HOMA-IR ( $\mu \mathrm{U} / \mathrm{mmol})$ & $2.50 \pm 1.91(2.61)$ & $2.45 \pm 1.79(1.93)$ & ${ }^{\mathrm{a}} 0.144$ \\
\hline HbA1c (\%) & $5.55 \pm 0.42$ & $5.47 \pm 0.42$ & ${ }^{\mathrm{b}} \mathbf{0 . 3 9 7}$ \\
\hline Glucose (mg/dL) & $96.13 \pm 9.88$ & $93.97 \pm 10.33$ & ${ }^{\mathrm{b}} 0.345$ \\
\hline Urea $(\mathrm{mg} / \mathrm{dL})$ & $24.00 \pm 2.85(33.55)$ & $24.98 \pm 6.58(23.70)$ & ${ }^{\mathrm{a}} 0.001 * *$ \\
\hline Creatinine $(\mathrm{mg} / \mathrm{dL})$ & $0.92 \pm 0.37(0.79)$ & $0.64 \pm 0.09(0.64)$ & ${ }^{\mathrm{a}} 0.001 * *$ \\
\hline Uric acid (mg/dL) & $4.94 \pm 2.01$ & $4.10 \pm 0.94$ & ${ }^{\mathrm{b}} 0.014 *$ \\
\hline AST (U/L) & $19.95 \pm 6.09(16.90)$ & $18.63 \pm 6.79(16.00)$ & a 0.773 \\
\hline ALT (U/L) & $17.65 \pm 8.18(13.50)$ & $16.86 \pm 8.10(14.00)$ & ${ }^{\mathrm{a}} \mathbf{0 . 3 7 9}$ \\
\hline LDH (U/L) & $183.48 \pm 28.59(186.00)$ & $191.18 \pm 28.89(186.00)$ & ${ }^{\mathrm{a}} 0.456$ \\
\hline $\mathrm{TG}(\mathrm{mg} / \mathrm{dL})$ & $134.04 \pm 66.17(134.50)$ & $122.44 \pm 92.35(93.55)$ & ${ }^{\mathrm{a}} 0.132$ \\
\hline Total cholesterol (mg/dL) & $212.41 \pm 52.35$ & $197.50 \pm 42.96$ & ${ }^{\mathrm{b}} 0.185$ \\
\hline HDL-c (mg/dL) & $55.89 \pm 13.1$ & $59.12 \pm 17.11$ & ${ }^{\mathrm{b}} \mathbf{0 . 3 4 3}$ \\
\hline LDL-c (mg/dL) & $133.62 \pm 47.36$ & $115.23 \pm 36.41$ & ${ }^{\mathrm{b}} 0.067$ \\
\hline $\mathrm{Ca}(\mathrm{mg} / \mathrm{dL})$ & $9.54 \pm 0.56$ & $9.30 \pm 0.51$ & ${ }^{\mathrm{b}} 0.054$ \\
\hline $\mathrm{Na}(\mathrm{mEq} / \mathrm{L})$ & $138.12 \pm 20.00(141.00)$ & $140.33 \pm 1.59(140.00)$ & ${ }^{\mathrm{a}} 0.158$ \\
\hline$K(m E q / L)$ & $4.45 \pm 0.43$ & $4.41 \pm 0.42$ & ${ }^{\mathrm{b}} 0.698$ \\
\hline Total protein (g/dL) & $7.33 \pm 0.45$ & $7.17 \pm 0.34$ & ${ }^{\mathrm{b}} 0.088$ \\
\hline Albumin $(g / d L)$ & $4.47 \pm 0.33$ & $4.48 \pm 0.29$ & ${ }^{\mathrm{b}} 0.858$ \\
\hline TSH (mU/L) & $2.00 \pm 0.99(1.72)$ & $3.30 \pm 3.43(2.10)$ & ${ }^{\mathrm{a}} 0.147$ \\
\hline Insulin (mU/L) & $11.85 \pm 7.40(10.81)$ & $10.60 \pm 8.15(8.46)$ & a 0.199 \\
\hline WBC $\left(/ \mathrm{mm}^{3}\right)$ & $6.95 \pm 1.75(6.79)$ & $7.00 \pm 2.14(6.98)$ & 1.000 \\
\hline HGB (g/dL) & $12.65 \pm 1.22$ & $12.82 \pm 1.14$ & ${ }^{\mathrm{b}} 0.535$ \\
\hline НСТ (\%) & $39.05 \pm 3.74$ & $39.68 \pm 3.31$ & ${ }^{\mathrm{b}} 0.433$ \\
\hline PLT $\left(/ \mathbf{m m}^{3}\right)$ & $250.24 \pm 66.55$ & $258.82 \pm 60.10$ & ${ }^{\mathrm{b}} 0.555$ \\
\hline MPV (fL) & $8.14 \pm 1.36$ & $8.39 \pm 1.91$ & ${ }^{\mathrm{b}} \mathbf{0 . 4 9 0}$ \\
\hline Urine density $\left(\mathrm{kg} / \mathrm{cm}^{3}\right)$ & $1013.31 \pm 7.83$ & $1014.61 \pm 6.40$ & ${ }^{\mathrm{b}} 0.435$ \\
\hline Urine protein $(\mathrm{mg} / \mathrm{dL})$ & $0.24 \pm 0.58(0)$ & $0.10 \pm 0(0)$ & ${ }^{\mathrm{a}} 0.022 * *$ \\
\hline Spot creatinine $(\mathrm{mg} / \mathrm{dL})$ & $122.81 \pm 74.69$ & $131.43 \pm 67.34$ & ${ }^{\mathrm{b}} 0.597$ \\
\hline Urine MA/KR (mg/g) & $90.37 \pm 178.73(18.05)$ & $5.78 \pm 5.67(3.90)$ & ${ }^{\mathrm{a}} 0.001 * *$ \\
\hline Urine microalbumin (mg/day) & $8.65 \pm 14.75(1.76)$ & $0.78 \pm 0.84(0.45)$ & ${ }^{\mathrm{a}} 0.001 * *$ \\
\hline Obesity degree (\%) & $24.25 \pm 16.95(19.21)$ & $14.22 \pm 7.68(15.85)$ & ${ }^{\mathrm{a}} 0.017 *$ \\
\hline Fat $(\mathrm{kg})$ & $24.69 \pm 10.26$ & $22.56 \pm 4.90$ & ${ }^{\mathrm{b}} 0.232$ \\
\hline Fat $(\%)$ & $32.98 \pm 9.04$ & $33.16 \pm 4.72$ & ${ }^{\mathrm{b}} 0.912$ \\
\hline Body impedance (ohm) & $576.36 \pm 79.17$ & $599.58 \pm 54.62$ & ${ }^{\mathrm{b}} 0.153$ \\
\hline Body muscle (kg) & $25.52 \pm 3.28$ & $24.42 \pm 1.80$ & ${ }^{\mathrm{b}} \mathbf{0 . 0 8 6}$ \\
\hline Body fat (kg) & $11.37 \pm 5.65$ & $10.40 \pm 3.20$ & ${ }^{\mathrm{b}} \mathbf{0 . 3 4 1}$ \\
\hline Metabolic age (years) & $50.66 \pm 16.42$ & $47.64 \pm 6.52$ & ${ }^{\mathrm{b}} 0.272$ \\
\hline Liquid $(\mathbf{k g})$ & $34.65 \pm 3.73$ & $32.78 \pm 2.26$ & ${ }^{\mathrm{b}} 0.008 * *$ \\
\hline Liquid (\%) & $49.01 \pm 6.65$ & $48.90 \pm 3.44$ & ${ }^{\mathrm{b}} 0.921$ \\
\hline
\end{tabular}

aMann-Whitney U test; ' Student's $t$-test; ${ }^{*} P<0.05 ;{ }^{*} P<0.01$. MDRD, modification of diet in renal diseases; HOMA-IR, homeostasis model assessment of insulin resistance; AST, aspartate aminotransferase; ALT, alanine aminotransferase; LDH, lactate dehydrogenase; TG, triglycerides; HDL-c, high-density lipoprotein cholesterol; LDL-c, low-density lipoprotein cholesterol; TSH, thyroid-stimulating hormone; WBC, white blood cell count; HGB, hemoglobin; HCT, hematocrit; PLT, platelet; MPV, mean platelet volume. 
No significant difference was observed between male patients and controls and female patients and controls in terms of body fat and liquid distribution $(P>0.05)$ (Table 6).

\section{DISCUSSION}

The present study indicated that patients with PKD have similar resistance to insulin action when compared to healthy population probably depending on the similar body fat distribution. Patients with PKD have higher level of blood pressure and uric acid and microalbuminuria independent from body fat distribution.

There is an increasing trend of high BMI among patients with ADPKD from 1985 to 2002. ${ }^{[10]}$ According to a Polish study, the incidence of metabolic syndrome among patients with ADPKD was $14 \%$, while it was $33 \%$ for DM in the study from United States. ${ }^{[10,11]}$ CVD is still the most common cause of death in ADPKD ${ }^{[12]}$. ADPKD is a systemic disease involving multiple organs and affecting metabolic parameters which make patients with ADPKD candidates of metabolic disorders and CVDs.

Memili et al. pointed out the relation between ADPKD and insulin resistance independent from preserved renal functions. ${ }^{[13]}$ Patients with ADPKD with well renal reserve have impaired insulin tolerance test. Turkmen $e t$ al. showed that IR accompanies ADPKD at the earlier stages of disease ${ }^{[14]}$. Nowacka et al. found an increased rate of IR among patients with ADPKD, especially carriers of some gene mutations. ${ }^{[1]]}$ Reed et al. revealed out a significant association of hyperinsulinemia, decreased insulin sensitivity, and type 2 diabetes with renal cyst volumes. ${ }^{[10]}$ Also diabetic patients with ADPKD die at a younger age when compared to nondiabetic counterparts with better metabolic control. ${ }^{[10]}$ Contradictorily, Vandana et al. failed to determine the association between IR and ADPKD ${ }^{[15]}$. Similarly, we did not examine a relationship.

Increased adipose tissue is defined as lipid ratio of $>19 \%$ for men and $22 \%$ for women. ${ }^{[16]}$ Several methods were used to assess the obesity degree and the distribution of fat and muscle mass. Anthropometric measurements are useful and noninvasive tools to examine the nutritional status as well as the body fat distribution. These measurement include weight, height, BMI, waist-hip circumference and ratio, calf circumference, overarm length, skinfold thickness, and bioelectrical impedance.

A well-known correlation has been established between body weight and blood pressure. A study by Zhang et al. showed that blood pressure control is poorly achieved in patients with BMI of $>30 \mathrm{~kg} / \mathrm{m}^{2}$ when compared to nonobese individuals. ${ }^{[17]} \mathrm{A}$ weight gain of $5-10$ and $>25$ $\mathrm{kg}$ have 1.7- and 5.2-fold increased risk of hypertension, respectively. ${ }^{[18]}$ On the other hand, body fat distribution, especially abdominal obesity, is associated with higher risk of hypertension, ${ }^{[19]}$ Patients with ADPKD with DM or MS have earlier age at the diagnosis of hypertension and are associated with poorly controlled blood pressure (Reed). Seeman $e t$ al. determined a significant correlation between systolic or diastolic blood pressure and the number of and volume of renal cysts. ${ }^{[20]}$

Helal et al. determined that serum uric acid level is associated with the earlier onset of hypertension and increased risk of ESRD independent of BMI and renal functions. ${ }^{[2]}$ Hyperuricemia is an established contributor of decreased blood flow as a result of endothelial dysfunction. Hyperuricemia causes decreased nitric oxide production and increased vascular smooth muscle proliferation, which are associated with endothelial dysfunction. ${ }^{[2]}$ Although hyperuricemia was associated with reduced eGFR, it

\begin{tabular}{|c|c|c|c|c|c|}
\hline & & & \multirow{2}{*}{$\frac{\text { Patients }}{n(\%)}$} & \multirow{2}{*}{$\begin{array}{l}\text { Controls } \\
n(\%)\end{array}$} & \multirow{2}{*}{$P$} \\
\hline & & & & & \\
\hline \multirow[t]{6}{*}{ Male } & Fat & $<20 \%$ & $17(50)$ & $12(50)$ & 0.150 \\
\hline & & $20-27 \%$ & $10(29.4)$ & $11(45.8)$ & \\
\hline & & $>27 \%$ & 7 (20.6) & $1(4.2)$ & \\
\hline & Liquid & $<50 \%$ & $2(5.9)$ & $0(0)$ & 0.476 \\
\hline & & $50-60 \%$ & $18(52.9)$ & $14(58.3)$ & \\
\hline & & $>60 \%$ & $14(41.2)$ & $10(41.7)$ & \\
\hline \multirow[t]{6}{*}{ Female } & Fat & $<20 \%$ & $5(11.4)$ & $0(0)$ & 0.124 \\
\hline & & $20-27 \%$ & $6(13.4)$ & $4(12.1)$ & \\
\hline & & $>27 \%$ & $33(75)$ & 29 (87.9) & \\
\hline & Liquid & $<50 \%$ & $25(56.8)$ & $23(69.7)$ & 0.228 \\
\hline & & $50-60 \%$ & $16(36.4)$ & $10(30.3)$ & \\
\hline & & $>60 \%$ & $3(6.8)$ & $O(0)$ & \\
\hline
\end{tabular}

Chi-square test; body fat ratio: $<20 \%$ low, 20-27\% normal, $>27 \%$ high; body liquid ratio: $<50 \%$ low, 50-60\% normal, $>60 \%$ high. 
was not an independent factor for renal progression in ADPKD ${ }^{[23]}$ Uric acid level is maintained in normal range in individuals with ADPKD with normal renal function. Hyperuricemia and decreased renal excretion of uric acid occur as a result of renal function worsening. ${ }^{[2]}$

Positive correlation between lipid parameters and BMI or CV mortality was established previously. ${ }^{[25]}$ LDL level is higher than normal in $83 \%$ of female and $57 \%$ of male individuals with BMI of $>25 \mathrm{~kg} / \mathrm{m}^{2} \cdot{ }^{[26]}$ A decrease of $1 \%$ in LDL is resulted with $2 \%$ decrease of coronary heart disease. ${ }^{[27]}$ Abdominal, waist, and hip fat distribution is associated with increased LDL-to-HDL ratio and triglyceride level, which are worse parameters of metabolic control. ${ }^{[28]}$

Obese individuals have higher incidence of microalbuminuria and proteinuria. ${ }^{[29]}$ Obesity is a well-known risk factor of glomerular disorders. Memili et al. found no significant relation between BMI and ESRD. ${ }^{[13]}$ Tirosh et al. showed that weight loss improves renal functions ${ }^{[30]}$. Because approximately 600,000 Americans are affected with ADPKD and more than 2,000 patients are starting dialysis every year, early interventions to slow down the progress are essential in $\mathrm{ADPKD}^{[31]}$.

Low sample size and single point measurement of variables were two major limitations of the study. A number of patients with PKD who have exclusion criteria mentioned in Patients and methods section were excluded. Crosssectional design of the study was another limitation. The importance of the study was; to the best of our knowledge, this was the first report that indicates the association of body fat distribution and insulin resistance in patients with PKD. An interventional study to determine the effect of weight loss on IR in ADPKD may clarify these results.

CVD-related deaths account the most common cause of mortality in ADPKD. There is a strict relationship between IR and CV morbidity. ${ }^{[32]} \mathrm{CV}$ events may reversed by early interventions to decrease IR. Physical exercise and life style modifications are recommended to overwhelm CV complications. Further studies with larger sample size will help better understanding of IR in patients with PKD.

\section{Conflict of Interest}

The authors declare that there is no conflict of interest.

\section{REFERENCES}

1. Germino GG, Chapman AB. Autosomal Dominant Polycystic Kidney Disease. In: Scriver CS, Baudet AL, Sly WS, Vale D, Childs B, Kinzler KW, Volgelstein B (eds). The Metabolic and Molecular Basis of inherited Disease. McGraw-Hill, New York 2001: 5467-89.

2. Fick-Brosnahan GM, Ecder T, Schrier RW. Polycystic kidney disease.
In: Schrier RW (ed), Diseases of the kidney an Urinary Tract (7th ed), Lippincott, Williams \&Wilkins, Philadelphia 2001: 547-88.

3. Lim SC, Tan BY, Chew SK, Tan CE. The relationship between insulin resistance and cardiovascular risk factors in overweight/obese nondiabetic Asian adults: the 1992 Singapore National Health Survey. Int J Obes Relat Metab Disord 2002; 26: 1511-6.

4. Vareesanggthipp K, Tong P, Wilkinson R, Thomas TH. Insulin resistance in adult polycystic kidney disease. Kidney Int 1997; 52: 503-8.

5. Hjelmeaeth J, Hartmann A. Insulin resistance in patients with adult polycystic kidney disease. Nephrol dial Transplant 1999; 14: 2521-2.

6. Kahn CR, Weir GC, King GL, Jacobson AM, Moses AC, Smith RJ. Joslin's Diabetes Mellitus, in Hawkins M and Rossetti L: Insulin resistance and its role in the pathogenesis of type II diabetes. Philadelphia, PA, Lippincott Williams \& Wilkins 2005; 425-48.

7. Clinical Guidelines on the Identification, Evaluation and Treatment of Overweight and Obesity in Adults-The evidence report. National Institutes of health. Obes Res 1998;6:51-209.

8. Kopelman PG, Stock MJ. Clinical obesity: Oxford. Blackwell Science 1998.

9. Matthews DR, Hosker JP, Rudenski AS, Naylor BA, Treacher DF, Turner RC. Homeostasis model assessment: insulin resistance and beta-cell function from fasting plasma glucose and insulin concentrations in man. Diabetologia 1985; 28: 412-9.

10. Reed B, Helal I, McFann K, Wang W, Yan XD, Schrier RW. The impact of type II diabetes mellitus in patients with autosomal dominant polycystic kidney disease. Nephrol Dial Transplant 2012; 27: 2862-5.

11. Pietrzak-Nowacka M, Safranow K, Byra E, Bińczak-Kuleta A, Ciechanowicz A, Ciechanowski K. Metabolic syndrome components in patients with autosomal-dominant polycystic kidney disease. Kidney Blood Press Res 2009; 32: 405-10.

12. Fick GM, Johnson AM, Hammond WS, Gabow PA. Causes of death in autosomal dominant polycystic kidney disease. J Am Soc Nephrol 1995; 5: 2048-56.

13. Memili VK. The relation of insulin resistance and renal failure in Autosomal polycystic kidney disease. Speciality Thesis. İstanbul, 2007.

14. Turkmen K, Tufan F, Alpay N, Kasikcioglu E, Oflaz H, Ecder SA, et al. Insulin resistance and coronary flow velocity reserve in patients with autosomal dominant polycystic kidney disease. Intern Med J 2012; 42 : 146-53.

15. Menon V, Rudym D, Chandra P, Miskulin D, Perrone R, Sarnak M. Inflammation, Oxidative Stress, and Insulin Resistance in Polycystic Kidney Disease. Clin J Am Soc Nephrol 2011; 6: 7-13.

16. Wang T, Ma X, Peng D, Zhang R, Sun X, Chen M, et al. Effects of Obesity Related Genetic Variations on Visceral and Subcutaneous Fat Distribution in a Chinese Population. Sci Rep 2016; 6: 20691.

17. Zhang YX, Wang SR, Zhao JS, Chu ZH. Prevalence of overweight and central obesity and their relationship with blood pressure among college students in Shandong, China. Blood Press Monit 2016 Apr 15. [Epub ahead of print]

18. Parker ED, Sinaiko AR, Kharbanda EO, Margolis KL, Daley MF, Trower NK, et al. Change in Weight Status and Development of Hypertension. Pediatrics 2016; 137: 1-9.

19. Lee J, Kim HJ, Cho B, Park JH, Choi HC, Lee CM, et al. Abdominal Adipose Tissue was Associated with Glomerular Hyperfiltration among Non- Diabetic and Normotensive Adults with a Normal Body Mass Index. PLoS One 2015; 10: e0141364.

20. Seeman T, Sikut M, Konrad M, Vondrichová H, Janda J, Schärer K. Blood pressure and renal function in autosomal dominant polycystic kidney disease. Pediatr Nephrol 1997; 11:592-6.

21. Helal I, Reed B, Schrier RW. Emergent early markers of renal progression in autosomal-dominant polycystic kidney disease patients: implications for prevention and treatment. Am J Nephrol 2012; 36: 162-7.

22. Cameron JS, Simmonds HA. Hereditary hyperuricemia and renal disease. Semin Nephrol 2005; 25: 9-18. 
23. Han M, Park HC, Kim H, Jo HA, Huh H, Jang JY, Kang AY, et al. Hyperuricemia and deterioration of renal function in autosomal dominant polycystic kidney disease. BMC Nephrol 2014; 15: 63.

24. William D. Kaehny. Uric acid handling in autosomal dominant polycystic kidney disease with normal filtration rates. Am J Med 1990; 49-52.

25. Srikanthan P, Horwich TB, Tseng CH. Relation of Muscle Mass and Fat Mass to Cardiovascular Disease Mortality. Am J Cardiol 2016; 117: 1355-60.

26. Khaodhiar L, Blackburn GL. Results of Expert Meetings: Obesity and Cardiovascular Disease: Obesity assessment. Am Heart J 2001; 142: 1095-101.

27. Unni SK, Quek RG, Biskupiak J, Lee VC, Ye X, Gandra SR. Assessment of statin therapy, LDL-C levels, and cardiovascular events among high-risk patients in the United States. J Clin Lipidol 2016; 10: 63-71.

28. Maffeis C, Schutz Y, Fornari E, Marigliano M, Tomasselli F, Tommasi $\mathrm{M}$, et al. Bias in food intake reporting in children and adolescents with type 1 diabetes: the role of body size, age and gender. Pediatr Diabetes 2016 Mar 16. [Epub ahead of print]
29. Esen B, Atay AE, Gunoz N, Gokmen ES, Sari H, Cakir I, et al. The relation of mean platelet volume with microalbuminuria and glomerular filtration rate in obese individuals without other metabolic risk factors: the role of platelets on renal functions. Clin Nephrol 2015; 83: 322-9.

30. Tirosh A, Golan R, Harman-Boehm I, Henkin Y, Schwarzfuchs D, Rudich A, et al. Diabetes Care 2013; 36: 2225-32.

31. Helal I, Reed B, Mettler P, Mc Fann K, Tkachenko O, Yan XD, et al. Prevalence of cardiovascular events in patients with autosomal dominant polycystic kidney disease. Am J Nephrol 2012; 36: 362-70.

32. Lumiaho A, Pihlajamäki J, Hartikainen J, Ikäheimo R, Miettinen R, Niemitukia $\mathrm{L}$, et al. Insulin resistance is related to left ventricular hypertrophy in patients with polycystic kidney disease type 1 . Am J Kidney Dis 2003; 41: 1219-24.

How to cite this article: Esen B, Gokmen ES, Kaya M, Ozkan B, Atay AE. The relation of anthropometric measurements and insulin resistance in patients with polycystic kidney disease. J TransI Intern Med 2016; 4: 127-34. 